\title{
Les petits exploitants agricoles à l'école inclusive : cas de l'apprentissage collectif par la vidéo et la radio sur la post-récolte du riz local au Bénin
}

\author{
Espérance ZOSSOU ${ }^{1,2 *}$, Seth GRAHAM-ACQUAAH ${ }^{2,4}$, John MANFUL ${ }^{3,4}$, \\ Simplice D. VODOUHE ${ }^{1}$ et Rigobert C. TOSSOU ${ }^{1}$ \\ ${ }^{1}$ Laboratoire de Sociologie et de Vulgarisation Rurales, Faculté des Sciences Agronomiques de l'Université \\ d'Abomey-Calavi, 01 BP 526 Cotonou, Bénin. \\ ${ }^{2}$ University of Arkansas, Fayetteville, AR, USA. \\ ${ }^{3}$ Ministry of Food and Agriculture (MOFA), Ghana. \\ ${ }^{4}$ Previous affiliation: Africa Rice Center (AfricaRice), Cotonou, Benin. \\ *Auteur correspondant ; BP 388 Abomey-Calavi, Bénin ; E-mail : esperancezossou@gmail.com
}

\author{
Received: 14-05-2021 $\quad$ Accepted: 22-08-2021 $\quad$ Published: 31-08-2021
}

\section{RESUME}

En Afrique subsaharienne, les petits exploitants agricoles ruraux ont difficilement accès aux connaissances et informations actualisées afin d'améliorer leur revenu. Pour renforcer l'apprentissage collectif au sein des acteurs des chaînes de valeur du riz local blanc et étuvé, la vidéo et la radio ont été utilisées de façon participative. La présente étude visait à comprendre l'influence de cet apprentissage collectif sur (i) le changement des pratiques des transformations et (ii) la qualité du riz local. Des interviews et suivis hebdomadaires ont été réalisés entre 2009 et 2018 au sud et nord du Bénin auprès de 240 transformateurs choisis aléatoirement. Des échantillons de riz ont été également collectés pour des analyses de qualité au laboratoire. L'analyse des données a été faite avec les tests t de Student, Wilcoxon, ANOVA, et le modèle de régression de Poisson. Les résultats ont montré une amélioration des pratiques de $14 \% ; 23 \%$ et $58 \%$ respectivement dans les villages contrôles, radio et vidéo. Dans le processus d'amélioration des pratiques, les transformateurs ont renforcé leur créativité en développant des technologies appropriées à leur environnement ; ce qui a eu un impact positif sur les qualités physiques et de cuisson du riz. Dans un contexte de pauvreté, d'insécurité alimentaire, de ruralité et d'analphabétisme, l'apprentissage collectif par la vidéo et la radio locale se révèle être une opportunité pour le renforcement du système d'innovation agricole.

(C) 2021 International Formulae Group. All rights reserved.

Mots clés : Apprentissage agricole, Vidéo, Radio locale, Riz local, Bénin.

\section{Smallholder farmers at inclusive school: Lessons learnt from collective learning through video and radio on local rice processing in Benin}

\section{ABSTRACT}

In sub-Saharan Africa, rural smallholder farmers have limited access to timely and up-todate knowledge and information to improve their incomes. To strengthen collective learning among rice sector' stakeholders, videos and radio were participatory used. This study aims to assess the 
impact of this collective learning on (i) changing rice processors practices and (ii) the quality of local rice. Interviews and weekly follow-ups were conducted between 2009 and 2018 in southern and northern Benin with 240 randomly selected rice processors. Rice samples were also collected for quality analysis in the laboratory. Data analysis was done with Student's t-tests, Wilcoxon, one-way ANOVA, and the Poisson regression model. The results showed an improvement in rice processors practices of $14 \% ; 23 \%$ and $58 \%$ respectively in control, video and radio villages. During the improvement of their practices, rice processors have increased their creativity by developing appropriate technologies to their environment; and this has positively impact on the physical and cooking qualities of the rice. In a context of poverty, food insecurity, rurality and illiteracy, collective learning through video and radio seems to be an opportunity to strengthen the agricultural innovation system, and a booster for change through adoption and adaptation of innovation.

(c) 2021 International Formulae Group. All rights reserved.

Keywords: Agricultural learning, Video, Local Radio, Post-harvest, Local rice, Benin.

\section{INTRODUCTION}

L'approche « top-down » longtemps utilisée dans la vulgarisation agricole en Afrique Subsaharienne a été beaucoup critiquée et qualifiée d'inefficace et moins apte à faire face aux exigences dynamiques de l'agriculture (Hakiza et al., 2004 ; Aplogan et al., 2021 ; Zossou et al., 2021). Plusieurs approches participatives de développement agricole ont donc vu le jour et ont été expérimentées à différentes échelles dans plusieurs pays : l'école champ paysan 'Farmer Field School' (FFS), l'apprentissage participatif et recherche action (APRA), le conseil à l'exploitation agricole familiale (CEF), etc. Ces approches participatives mettent un accent particulier sur la valorisation des connaissances et savoirs des populations locales et leur combinaison avec la connaissance scientifique pour améliorer l'apprentissage réciproque (Axinn, 1997 ; Struif-Bontkes et al., 2003). Malgré leur efficacité prouvée (Pretty et Vodouhe, 2001 ; Nederlof et Odonkor, 2006 ; Mancini et al., 2007), ces approches participatives sont exigeantes en ressources humaines disponibles et qualifiées, et malheureusement touchent un effectif très limité d'agriculteurs (Van Mele et al., 2005). Face à cette contrainte, la vidéo d'apprentissage agricole "Farmer to Farmer vidéo" (FFV) a été développée dans l'optique d'atteindre une large audience tout en respectant le principe d'apprentissage constructiviste (Van Mele, 2006).

La FFV est développée selon une approche nommée « zooming-in, zooming-out
» qui s'inspire du processus et des résultats des approches participatives et inclusives. Elle commence par une large consultation des parties prenantes pour définir les besoins d'apprentissage. Les communautés concernées sont donc consultées et écoutées dans le but d'obtenir une meilleure compréhension au sujet de leurs idées, leurs connaissances, les innovations locales produites en ce qui concerne la thématique d'intérêt (zooming-in). Des vidéos sont ensuite produites en étroite collaboration avec ces communautés ciblées qui ont été engagées dans le processus de production des innovations. Ensuite les vidéos élaborées sont montrées à d'autres communautés partageant les mêmes préoccupations que celles des communautés impliquées dans la production des vidéos (zooming-out). Ce qui conduit à plus d'échanges et de nouveaux ajustements pour l'amélioration des vidéos (Van Mele, 2006).

Pour favoriser l'apprentissage collectif au sein des acteurs concernés par la postrécolte du riz au Bénin, la FFV a été utilisée pour la $1^{\text {ère }}$ fois en 2006 dans le département des collines. Les vidéos ont été produites sur des technologies améliorées des opérations post-récoltes du riz local qui ont été développées par Houssou et al. en 2004 ; et Houssou en 2005. Les contraintes post-récoltes préoccupent les communautés rizicoles au Bénin car malgré les progrès réalisés dans la production du riz local (superficies allouées et quantités produites en croissance continue), le produit final éprouve des difficultés pour bien se positionner sur le marché local (Zossou et 
al., 2009a). La FFV a été donc utilisée afin de répondre au défi majeur qui est de rendre les informations et les technologies développées accessibles à une large audience du groupe cible.

En plus de la FFV, la radio rurale a été également utilisée pour la $1^{\text {ère }}$ fois en 2007 afin de rendre accessible l'information sur le riz disponible au niveau de la FFV. En effet, dans le contexte béninois caractérisé par une forte ruralité et un faible taux d'alphabétisation, la radio rurale est reconnue comme l'un des mass médias les plus appropriés car elle diffuse des informations dans les langues locales (Chapman et al., 2003 ; Ramirez et Quarry, 2004 ; Zossou et al., 2015). Les vidéos ont été donc utilisées comme des supports à partir desquels des scripts radiophoniques ont été développés pour des émissions radiophoniques en langues locales. L'utilisation de la FFV et de la radio rurale constitue une approche innovante pour répondre aux défis qui s'imposent au système post-récolte du riz local au Bénin. La présente recherche vise à comprendre le rôle de la vidéo et de la radio rurale dans le processus d'apprentissage collectif des acteurs de la pot-récolte du riz local. Elle se propose donc d'analyser les changements dans les pratiques de transformation du riz local et l'influence sur la qualité du riz local avec l'introduction de la FFV et de la radio rurale.

\section{MATÉRIEL ET MÉTHODES Milieu d'étude}

L'étude a été conduite au nord et au sud du Bénin, avec six villages sélectionnés dans deux communes différentes au nord; et six villages sélectionnés dans trois communes différentes au sud. Au total, les données ont été collectées dans douze villages situés dans cinq communes différentes. Le choix des communes a été fait en se basant sur l'importance de la production du riz. Les villages ont été choisis aléatoirement tout en respectant une distanciation d'au moins $8 \mathrm{~km}$ afin d'éviter les échanges d'informations faciles entre villages voisins. Le choix des auxiliaires de vulgarisation agricole (FFV / radio / contrôle) a été fait de façon aléatoire (Tableau 1).

\section{Échantillonnage et collecte des données}

L'échantillon est constitué de 20 transformateurs de riz sélectionnés aléatoirement parmi ceux recensés dans chaque village (un minimum de 24 et un maximum de 45 transformateurs). $\mathrm{Au}$ total, 240 transformateurs de riz à raison de 120 au nord et 120 au sud du Bénin ont été enquêtés. En plus du questionnaire d'enquête, des fiches de suivi ont été utilisées de façon hebdomadaire pendant trois mois en pleine période postrécolte pour la triangulation des informations collectées à travers le questionnaire.

Parmi les 20 transformateurs de riz enquêtés dans chaque village, 3 ont été sélectionnés au hasard pour la collecte des échantillons de riz pour des analyses de qualité au laboratoire Qualité Grain du Centre du Riz pour l'Afrique (Africa Rice). Un total de 18 échantillons de riz étuvé au nord et de 18 échantillons de riz blanc au sud du Bénin ont été collectés pour des analyses de qualité au laboratoire avant l'apprentissage avec une approche méthodologique résumée dans le Tableau 2.

La méthodologie utilisée pour la collecte des données empiriques a été itérative avec quatre étapes principales à savoir : (i) la collecte des données en 2010 avant l'apprentissage ; (ii) l'expérimentation de l'apprentissage par la vidéo ou la radio en 2010 (iii) la collecte des données en 2012 soit deux ans après l'apprentissage ; et (iv) la collecte des données en 2018, soit huit ans après l'apprentissage. Des approches qualitatives et quantitatives ont été utilisées avec des techniques et outils tels que : focus-group, observation participante, entretiens semistructurés, fiches de suivi, et prélèvements d'échantillons de riz pour des analyses au laboratoire.

$\mathrm{Au}$ cours des séances d'apprentissage par la vidéo (FFV) et la radio en 2010, deux projections vidéo et émissions radiophoniques ont été faites dans les langues locales dans les villages retenus; une $1^{\text {ère }}$ en début de la campagne agricole et une $2^{\text {nde }}$ en début de la récolte (soit environ 6 mois d'intervalle). Les projections vidéo ont été facilitées par les agents des services publics de vulgarisation agricole. La $1^{\text {ère }}$ vidéo est relative à la transformation à petite échelle du riz blanc et 
explique les différentes étapes à suivre de la récolte jusqu'au décorticage et au stockage. La seconde vidéo explique les différentes étapes à suivre pour étuver le riz local avec un matériel amélioré. En ce qui concerne les émissions radiophoniques, elles ont été réalisées sur la base des scripts radiophoniques tirés des deux vidéos citées plus haut. Le type de l'émission (directe, interactive ou différée), la durée, l'association ou non d'une personne ressource (des centres agricoles, des organisations paysannes, etc.) ont été laissés à l'initiative des animateurs des radios rurales concernées. Les animateurs des radios rurales ont bénéficié des supports de $\mathrm{CD}$ vidéo à partir desquels des scripts radiophoniques ont été développés. Ce qui a contribué au renforcement de leurs capacités dans le domaine avant l'animation des émissions radiophoniques. En plus de l'apprentissage par la vidéo et la radio locale, le matériel amélioré d'étuvage de riz a fait l'objet d'un don juste après l'apprentissage dans les villages où l'étuvage du riz se fait.

Le matériel amélioré d'étuvage du riz est une technologie développée par la recherche avec la participation active des femmes étuveuses du riz au Bénin (FAO, 1997; Houssou et al., 2004). Il est composé d'un bac en tôle galvanisé, perforé (à la base et au tiers inférieur) et adapté à une marmite en fonte d'aluminium. Ce dispositif de pré-cuisson du riz paddy à la vapeur vise à améliorer de façon quantitative et qualitative le rendement du riz étuvé tout en maintenant ses qualités organoleptiques et sa valeur marchande.

Deux ans après l'apprentissage par la vidéo et la radio, les mêmes données quantitatives et qualitatives (données sociodémographiques, pratiques de transformation du riz de la récolte au décorticage, adoption et ou adaptation des technologies vulgarisées, mode de transaction, prix de vente, circuits de commercialisation...) collectées en 2010 avant l'apprentissage ont été collectées à nouveau auprès des mêmes transformateurs de riz. Les 18 échantillons de riz ont été à nouveau collectés chez les mêmes transformateurs pour les mêmes analyses au laboratoire.

En 2018, huit ans après l'apprentissage en 2010, des données sur les pratiques de transformation du riz paddy ont été à nouveau collectées auprès des mêmes transformateurs, afin d'apprécier la durabilité dans les changements observés en 2012. Les données ont été collectées deux ans et huit ans après les premières expérimentations afin de mesurer l'impact de l'apprentissage à court et à long terme. Les effectifs des enquêtés ont varié entre 2010 et 2018 passant de 240 en 2010 à 231 en 2012 et à 228 en 2018 à cause des cas de décès et de déplacements (Tableau 1).

\section{Traitement et analyse des données}

La revue de littérature fait ressortir six étapes principales dans la transformation à petite échelle du paddy en riz blanc et en riz étuvé de bonne qualité au Bénin (Houssou et al., 2004). Au cours de la collecte des données sur les pratiques de transformation du riz local, des scores ont été attribués (0 à 2$)$ à chacune des six étapes de chaque type de transformation (0 si le transformateur ne respecte pas les recommandations de l'étape ; 1 s'il respecte parfois, et 2 s'il respecte toutes les fois). Une somme a été faite pour les six étapes principales pour un score par transformateur enquêté. Au cours du traitement des données, une catégorisation a été faite par type de transformation selon les scores obtenus par les transformateurs :' $0 \leq$ score $\leq 4$ ' = 'pratiques non recommandées' ; ' $4<$ score $\leq 8^{\prime}$ ' 'pratiques peu recommandées' et ' $8<$ score $\leq$ 12 ' = 'pratiques recommandées'.

Les données collectées ont été traitées et analysées avec les logiciels Excel, SPSS et STATA. La statistique descriptive (pourcentages, fréquences, moyennes) et des tableaux, graphes et histogrammes ont été utilisés. La méthode de double différence (Diff-in-Diff) a été utilisée pour faire une comparaison avant et après et avec et sans l'apprentissage avec le test de Wilcoxon au niveau de chaque groupe de transformateurs (contrôle, radio et vidéo) et le test ANOVA à un facteur entre les différents groupes. La régression de Poisson a été utilisée pour analyser les différents facteurs qui influencent l'amélioration des pratiques des transformateurs de riz. Ce modèle a été choisi parce que la variable dépendante (pratiques des transformateurs) est discrète (ne peut revêtir qu'un nombre défini de valeurs réelles) et positive. 
Tableau 1: Récapitulatif de l'échantillonnage par village et par type d'apprentissage au nord et au sud du Bénin.

\begin{tabular}{|c|c|c|c|c|c|c|c|c|c|c|c|c|}
\hline \multirow[t]{2}{*}{ Région } & \multirow[t]{2}{*}{ Commune } & \multirow[t]{2}{*}{ Village } & \multirow[t]{2}{*}{$\begin{array}{l}\text { Type } \\
\text { d'apprentissage }\end{array}$} & \multicolumn{3}{|c|}{$\begin{array}{l}\text { Effectifs des échantillons pour } \\
\text { les questionnaires }\end{array}$} & \multicolumn{3}{|c|}{$\begin{array}{l}\text { Effectifs des échantillons pour } \\
\text { les fiches de suivi }\end{array}$} & \multicolumn{3}{|c|}{$\begin{array}{l}\text { Effectifs des échantillons pour } \\
\text { l'analyse de laboratoire }\end{array}$} \\
\hline & & & & 2010 & 2012 & 2018 & 2010 & 2012 & 2018 & 2010 & 2012 & 2018 \\
\hline \multirow{6}{*}{$\begin{array}{l}\text { Nord du } \\
\text { Bénin }\end{array}$} & Malanville & Toumboutou & Contrôle & 20 & 20 & 20 & 20 & 20 & 20 & 3 & 3 & - \\
\hline & & Garou & Radio & 20 & 20 & 20 & 20 & 20 & 20 & 3 & 3 & - \\
\hline & & Bodjécali & FFV & 20 & 20 & 19 & 20 & 20 & 19 & 3 & 3 & - \\
\hline & Banikoara & Dêrou & Contrôle & 20 & 20 & 20 & 20 & 20 & 20 & 3 & 3 & - \\
\hline & & Kokiborou & Radio & 20 & 20 & 19 & 20 & 20 & 19 & 3 & 3 & - \\
\hline & & Kockey & FFV & 20 & 20 & 20 & 20 & 20 & 20 & 3 & 3 & - \\
\hline \multirow{6}{*}{$\begin{array}{l}\text { Sud du } \\
\text { Bénin }\end{array}$} & Covè & Lélé & Contrôle & 20 & 20 & 19 & 20 & 20 & 19 & 3 & 3 & - \\
\hline & & Lainta & Radio & 20 & 19 & 19 & 20 & 19 & 19 & 3 & 3 & - \\
\hline & & Koussin & FFV & 20 & 19 & 19 & 20 & 19 & 19 & 3 & 3 & - \\
\hline & Adjohoun & Mitro & Contrôle & 20 & 17 & 17 & 20 & 17 & 17 & 3 & 3 & - \\
\hline & & Houêda & Radio & 20 & 19 & 19 & 20 & 19 & 19 & 3 & 3 & - \\
\hline & & Djigbé & FFV & 20 & 17 & 17 & 20 & 17 & 17 & 3 & 3 & - \\
\hline TOTAL & & & & 240 & 231 & 228 & 240 & 231 & 228 & 36 & 36 & - \\
\hline
\end{tabular}


Tableau 2 : Récapitulatif de la méthodologie utilisée au laboratoire pour l'analyse de la qualité des échantillons de riz collectés.

\begin{tabular}{|c|c|c|}
\hline \multicolumn{2}{|c|}{ Caractéristiques } & \multirow{2}{*}{$\begin{array}{l}\text { Approche méthodologique } \\
\text { Un échantillon de } 20 \mathrm{~g} \text { de riz décortiqué est pesé en } 3 \text { répétitions de l'échantillon ramené du terrain. Les impuretés (corps étrangers, } \\
\text { grains brûlés, grains non décortiqués...) sont triées et pesées. Pourcentage d'impureté }=\text { poids des impuretés } \times 100 / 20 \mathrm{~g} \text {. }\end{array}$} \\
\hline Physiques & Pourcentage d'impuretés & \\
\hline & Taux de brisure & $\begin{array}{l}\text { Sur un échantillon de } 20 \mathrm{~g} \text { de riz prélevé (en } 3 \text { répétitions), les grains entiers sont séparés des grains brisés à l'aide d'un appareil } \\
\text { séparateur des grains 'test rice grader'. Taux de brisure = poids des grains brisés } / 20 \mathrm{~g}\end{array}$ \\
\hline & Dureté des grains & $\begin{array}{l}\text { La dureté du grain a été mesurée en utilisant un appareil testeur de dureté (LDT Fujihara Seisakusho, Japon). Pour chaque échantillon } \\
\text { de riz, } 10 \text { grains ont été testés en } 3 \text { répétitions. La valeur est indiquée au son de craquement par une aiguille. }\end{array}$ \\
\hline & Test de cœurs blancs & $\begin{array}{l}\text { Deux cents grains de riz entiers sont sélectionnés au hasard dans chaque échantillon et évalués visuellement pour détecter les cœurs } \\
\text { blancs (endospermes amidonnés). Un score de } 1 \text { est donné pour moins de } 10 \% \text { de cœurs blancs ; } 5 \text { pour } 10 \text { à } 20 \% \text { de cœurs blancs et } \\
9 \text { pour plus de } 20 \% \text { de cœurs blancs. Ceci est fait selon les mesures d'évaluation standard de l'Institut de Recherche International sur } \\
\text { le Riz (IRRI, 1996). }\end{array}$ \\
\hline & Test de couleur & $\begin{array}{l}\text { Un appareil 'Hunter } \mathrm{L} \text {, a, b color scale' est utilisé pour } 20 \mathrm{~g} \text { de riz (en } 3 \text { répétitions) prélevés dans les échantillons. Les valeurs } \mathrm{L} \text {, a } \\
\text { et b sont données par l'appareil. La couleur est donnée par la valeur } E=\sqrt{ }\left(\mathrm{L}^{2}+\mathrm{a}^{2}+\mathrm{b}^{2}\right) \text {. }\end{array}$ \\
\hline \multirow[t]{3}{*}{ Cuisson } & Temps de cuisson & $\begin{array}{l}\text { On prélève } 5 \mathrm{~g} \text { de chaque échantillon en } 3 \text { répétitions. Chaque quantité prélevée est versée dans } 135 \mathrm{ml} \text { d'eau distillée portée à } \\
\text { ébullition sur des plaques chauffantes. Après } 10 \text { minutes d'ébullition, } 10 \text { grains sont prélevés et pressés entre deux boîtes de Pétri. } \\
\text { Cette opération est répétée toutes les minutes et on considère le riz comme cuit lorsqu'on a au moins } 9 \text { des } 10 \text { grains complètement } \\
\text { écrasés. La durée du temps de cuisson est détectée à l'aide d'un chronomètre. }\end{array}$ \\
\hline & Elongation des grains & $\begin{array}{l}\text { On choisit aléatoirement } 10 \text { grains de chaque échantillon en trois répétitions. Après la mesure de leurs longueurs respectives, les } \\
\text { grains sont placés dans un cylindre à mailles métallique que l'on place dans } 135 \mathrm{ml} \text { d'eau préalablement portée à ébullition dans un } \\
\text { bécher. On laisse l'ensemble sur les plaques chauffantes durant le temps de cuisson déterminé au préalable. Après cuisson, les } \\
\text { longueurs des grains sont mesurées de nouveau. Le taux d'élongation = longueur moyenne des grains cuits/longueur moyenne des } \\
\text { grains non cuits. }\end{array}$ \\
\hline & $\begin{array}{l}\text { Augmentation de volume } \\
\text { et absorption d'eau }\end{array}$ & $\begin{array}{l}\text { On prélève } 8 \mathrm{~g} \text { de chaque échantillon en } 3 \text { répétitions qu'on met dans des cylindres à mailles métalliques. L'ensemble (cylindre et riz) } \\
\text { est pesé de nouveau et donne un poids } \mathrm{W} 1 \text { avec une hauteur } \mathrm{H} 1 \text {. Les cylindres contenant le riz sont placés chacun dans } 135 \mathrm{ml} \mathrm{d} \text { 'eau } \\
\text { préalablement portée à ébullition. On laisse chaque échantillon bouillir pendant la durée retenue pour sa cuisson. Les échantillons et } \\
\text { les cylindres à mailles sont ensuite laissés pendant } 2 \text { minutes afin de permettre à l'eau de s'écouler. Le poids après cuisson est pris } \\
\text { (W2) ainsi que la hauteur }(\mathrm{H} 2) \text {. Le taux moyen de l'absorption d'eau }=(\mathrm{W} 2-\mathrm{W} 1) / 8 \mathrm{~g} \text { et le taux d'augmentation de volume }=\mathrm{H} 2 / \mathrm{H} 1 \text {. }\end{array}$ \\
\hline
\end{tabular}




\section{RESULTATS}

\section{Profil des transformateurs et état des lieux des pratiques de transformation du riz local avant l'apprentissage collectif}

Les transformateurs de riz ont été en majorité des femmes (67\% dans l'ensemble de l'échantillon, $100 \%$ dans le nord et $34 \%$ dans le sud du Bénin). Environ 54\% des transformateurs du paddy en riz blanc enquêtés sont des femmes. En ce qui concerne le riz étuvé, environ $92 \%$ des enquêtés sont des femmes. La quasi-totalité des transformateurs sont mariés(es) (100\% des hommes et $92 \%$ des femmes) et analphabètes ( $87 \%$ des femmes et $53 \%$ des hommes). La taille des ménages est d'environ 8 personnes en moyenne. La religion dominante dans le nord est l'Islam (100\%) et le Christianisme dans le sud (62\%). L'activité prédominante des hommes est l'agriculture (95\%) et celle des femmes est le petit commerce incluant la transformation et la vente du riz local (63\%). Deux principales chaînes de valeur caractérisent la transformation du riz paddy en riz décortiqué au Bénin (Figure 1) : riz blanc et riz étuvé.

Les activités post-récoltes s'étalent généralement sur 3 à 6 mois dans le sud alors qu'elles s'étendent sur 9 à 12 mois dans le nord du Bénin. Cette différence est due au fait que les producteurs au nord ont la possibilité de produire du riz pluvial et du riz des bas-fonds. Le nombre d'années d'expérience des transformateurs est de 9,40 $( \pm 5,16)$ au sud et de 9,96 $( \pm 8,69)$ au nord du Bénin. Les transformateurs du riz paddy ont appris leur savoir-faire de différentes sources catégorisées en deux types principaux (Figure 2): (i) la source formelle qui regroupe les ONG locales, nationales et internationales ainsi que les centres nationaux de recherche et de vulgarisation agricole (29\%) et (ii) la source informelle qui regroupe la famille, les amis et les voisins $(71 \%)$. Les femmes sont plus représentées dans la source d'apprentissage informelle (Figure 2) qui prédomine dans le nord du Bénin où la totalité des transformateurs de riz sont des femmes. Les résultats des enquêtes en 2010 avant l'apprentissage ont montré que la majorité des transformateurs $(76 \%$ et $97 \%)$ ont des pratiques non recommandées respectivement pour la transformation du riz blanc et celle du riz étuvé.

\section{Amélioration des pratiques de transformation du riz blanc après l'apprentissage collectif}

Les améliorations notées dans les pratiques de transformation du riz blanc sont résumées dans le Tableau 3. Les résultats du test de Wilcoxon ont donné des probabilités inférieures à 0,05 pour la comparaison des pratiques entre " 2010 et 2012 '" et "2010 et 2018" au sein de chacun des trois groupes de villages (contrôle, FFV et radio) pour le riz blanc. Les résultats du test ne montrent pas de différence significative entre 2012 et 2018 . Il y a eu donc une amélioration des pratiques entre 2010 et 2012 dans les trois groupes de villages (FFV, radio et contrôle); et les améliorations notées ont été maintenues entre 2012 et 2018.

L'amélioration notée dans les villages contrôles confirme l'analyse de Delarue et Cochet (2011) sur le fait que les sociétés rurales ne sont pas immobiles dans le temps et ne doivent pas être considérées comme incapables de se transformer et d'évoluer hors projet ou intervention exogène. D'où l'importance de ne pas utiliser seulement l'indicateur d'impact avant et après projet comme base de calcul dans les mesures d'impact.

Le test ANOVA à un facteur, utilisé pour la comparaison entre les trois groupes de villages (FFV, radio et contrôle), a montré une différence très significative en 2012 et en 2018 entre les trois types d'apprentissage vidéo, radio et contrôle. Les pratiques des transformateurs du riz blanc en 2012 et en 2018 sont meilleures dans les villages FFV comparés aux villages radio. Elles sont également meilleures dans les villages radio comparés aux villages contrôles. Ces améliorations concernent certains points essentiels tels que : (i) éviter le mélange variétal; (ii) faire le battage à un endroit propre; (iii) faire le vannage à plusieurs reprises, (iv) faire le 
séchage du riz au soleil et à l'ombre et (v) éviter de faire le stockage du paddy ou du riz décortiqué directement sur le sol cimenté ou non.

\section{Amélioration des pratiques d'étuvage du riz après l'apprentissage collectif}

Le test de Wilcoxon (Tableau 3) a montré des différences significatives dans les pratiques au niveau de chacun des trois groupes de villages entre '2010 et 2012 ' et '2010 et $2018^{\prime}$; et aucune différence significative entre '2012 et 2018 '. Les pratiques d'étuvage du riz se sont donc améliorées après l'apprentissage et sont restées stables dans le temps entre 2012 et 2018. La faible amélioration observée dans les villages contrôles peut s'expliquer par le fait que sept transformateurs de riz, dans l'un des villages contrôles (Toumboutou) au nord du Bénin, ont fortuitement suivi les émissions radiophoniques. Ce qui a entraîné un changement positif dans leurs pratiques.

Le test d'ANOVA (Tableau 3) à un facteur a montré une différence significative dans les pratiques d'étuvage du riz entre les trois groupes de villages en 2012 et les trois groupes en 2018. Dans les villages contrôles en 2012 et en 2018, les transformatrices de riz étuvé ont majoritairement des pratiques peu recommandées. Par contre, dans les villages vidéo et radio en 2012 et en 2018, les transformatrices ont majoritairement des pratiques recommandées. L'amélioration des pratiques dans les villages vidéo et radio en 2012 et confirmée en 2018 se situe à plusieurs niveaux à savoir (i) l'utilisation du matériel amélioré d'étuvage du riz; (ii) l'adoption des innovations locales pour imiter la pré-cuisson du riz paddy à la vapeur et (iii) l'amélioration des pratiques de triage, lavage, trempage, séchage et stockage du riz paddy.

Consécutivement à l'apprentissage collectif, les taux d'utilisation du matériel amélioré d'étuvage du riz ont été respectivement de $77 \%$ et $33 \%$ en 2012 et $79 \%$ et $35 \%$ en 2018 respectivement dans les villages FFV et radio; et $0 \%$ pour les deux années dans les villages contrôles. Pour l'utilisation du matériel en 2012, les femmes ont instauré un système de location dont le coût varie entre $500 \mathrm{~F}$ et $1000 \mathrm{~F}$ CFA par jour. En 2018 , les deux villages du nord où il $\mathrm{y} a$ eu des projections vidéo ont pu acquérir chacun un nouveau matériel d'étuvage du riz avec les fonds de location instaurés en 2012. Ceci n'a pas été le cas dans le sud du Bénin où le même système de location du matériel d'étuvage avait été instauré en 2012. La raison qui explique cet état de chose est que l'activité d'étuvage du riz n'est pas très développée dans le sud et ne constitue donc pas une priorité pour l'union des producteurs de riz dont les responsables sont chargés de la gestion des frais issus de la location du matériel.

Les transformateurs qui ont compris l'importance de la pré-cuisson du paddy à la vapeur au cours de l'apprentissage, et qui n'arrivent pas à louer le matériel faute de moyens financiers ou de disponibilité du matériel, ont innové localement afin d'imiter le principe de pré-cuisson à la vapeur. Cette innovation locale consiste à perforer des bassines en métal servant d'ustensiles de cuisine pour l'étuvage du riz. Les taux d'innovation ont été en 2018 respectivement dans les villages FFV, radio et contrôle de $79 \%, 70 \%$ et $8 \%$. D'autres éléments importants dans l'amélioration des pratiques d'étuvage du riz concernent le triage, le lavage, le séchage et le stockage du paddy. En 2012, respectivement $87 \%$ et $78 \%$ des enquêtés dans les villages vidéo et radio ont mentionné avoir amélioré leurs pratiques de transformation après avoir acquis de nouvelles connaissances au cours de l'apprentissage en 2010. Les pratiques améliorées ont été maintenues dans le temps, jusqu'en 2018.

\section{Déterminants de l'amélioration des pratiques de transformation du riz local}

Le modèle de régression de Poisson a été utilisé pour apprécier les facteurs susceptibles d'influencer l'amélioration des pratiques des transformateurs du riz local (Tableaux 4 et 5). 
Le modèle fait ressortir quatre facteurs qui ont un effet positif significatif sur l'amélioration des pratiques de transformation du riz paddy en riz blanc: (i) l'apprentissage par la FFV ; (ii) la fréquence de rencontre avec les facilitateurs du développement rural ; (iii) l'accès au crédit formel, et (iv) l'accès au crédit informel. La durée de l'activité de transformation du riz au cours de l'année a par contre eu un effet négatif significatif sur l'amélioration des pratiques de transformation du riz blanc.

Quant à l'amélioration des pratiques d'étuvage du riz, trois facteurs ont un effet positif significatif : (i) l'apprentissage par la FFV ; (ii) l'apprentissage par la radio et (iii) le type de transformation (c'est-à-dire la transformation du riz paddy en riz étuvé seul ou la transformation du riz paddy en riz étuvé et en riz blanc).

\section{Amélioration des caractéristiques physiques du riz local suite à l'apprentissage collectif}

Les caractéristiques physiques du riz blanc et étuvé portent sur le pourcentage d'impuretés, le taux de brisure, la forme et la dureté des grains, la présence de cœur blanc et la couleur des grains. Les valeurs des différentes caractéristiques sont résumées dans le Tableau 6. Entre 2010 et 2012, le pourcentage d'impuretés a augmenté de façon significative seulement dans les villages contrôles pour le cas du riz étuvé avec le test $t$ de Student. En 2012, le test ANOVA à un facteur utilisé pour une comparaison entre les trois groupes de villages, montre une différence significative pour le cas du riz étuvé.

Le test ANOVA à un facteur a montré une différence significative entre les taux de brisure des échantillons de riz étuvé des trois groupes de villages en 2012 ; avec un taux élevé dans les villages contrôles. De même, le test $\mathrm{t}$ de Student montre une régression significative du taux de brisure entre 2010 et 2012 dans les villages FFV et une augmentation significative dans les villages contrôles.
Le test $\mathrm{t}$ de Student a montré une différence significative entre les valeurs de la dureté des grains des échantillons de riz blanc et étuvé collectés entre 2010 et 2012 dans chacun des trois groupes de villages; avec de meilleurs scores dans les villages FFV et radio. Le test $\mathrm{t}$ de Student sur les valeurs de la présence de cœurs blancs a également montré une différence significative entre 2010 et 2012 dans les villages radio avec un faible score en 2012. En 2012, de meilleurs scores ont été enregistrés pour la couleur dans les villages FFV et radio.

\section{Amélioration des caractéristiques de cuisson du riz local suite à l'apprentissage collectif}

Les caractéristiques de cuisson du riz blanc et étuvé portent sur l'élongation, l'absorption d'eau, l'augmentation de volume et le temps de cuisson des grains de riz. Les valeurs relatives de ces différentes caractéristiques sont résumées dans le Tableaux 7. Les résultats ont montré au niveau de l'élongation une différence significative entre les échantillons de riz étuvé collectés entre 2010 et 2012 dans les villages FFV ; avec une valeur plus élevée pour les échantillons de 2012. Aussi existe-t-il une différence significative entre les échantillons de riz étuvé collectés dans les trois groupes de villages en 2012 ; avec des valeurs plus élevées dans les villages vidéo et radio. Les analyses de laboratoire ont montré qu'il existe une différence significative au niveau inter-villages et intra-village en ce qui concerne l'augmentation de volume des échantillons de riz blanc. En ce qui concerne l'absorption d'eau, les résultats ne permettent pas de tirer de bonnes conclusions et nécessitent donc plus d'investigations pour des études ultérieures.

Il n'existe pas de différence significative entre les échantillons de riz de 2012 des trois groupes de villages en termes de durée de cuisson. On observe cependant une différence significative entre 2010 et 2012 dans chacun des trois groupes de villages pour le cas du riz blanc avec des valeurs plus élevées en 2012 dans les villages vidéo. 


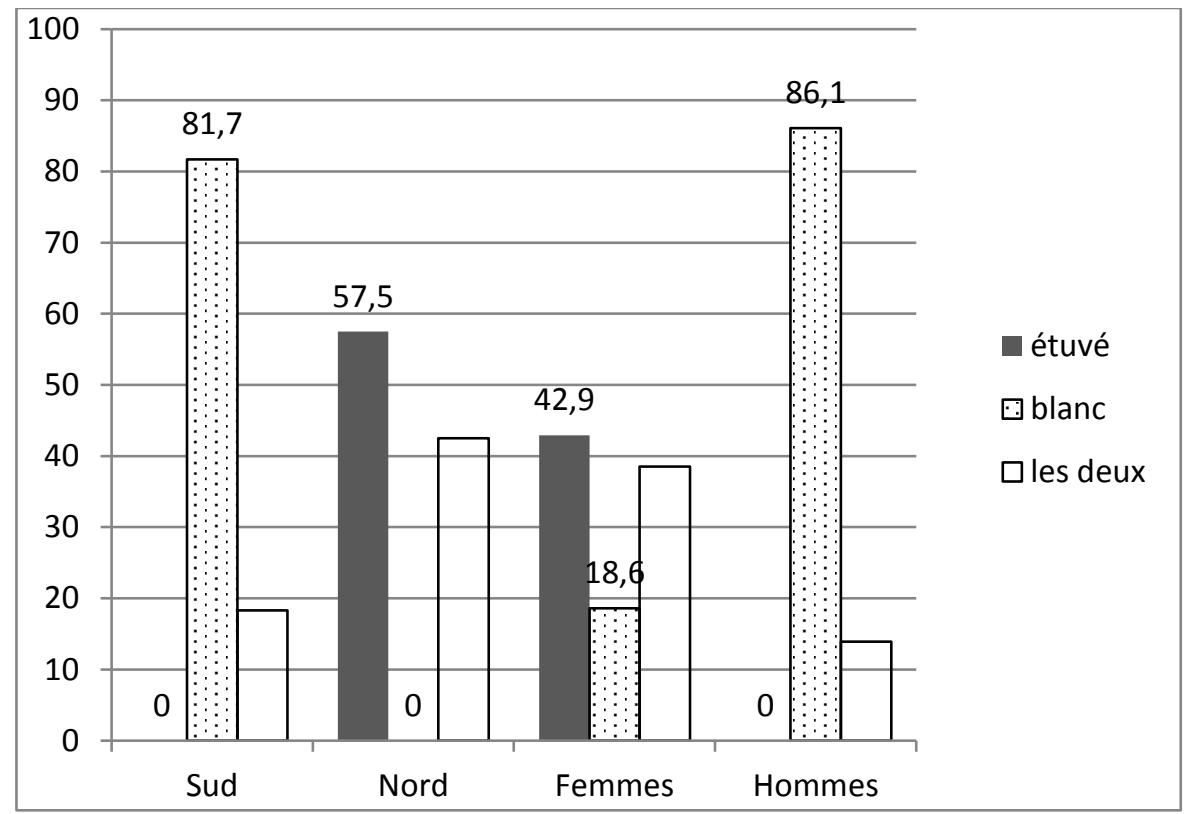

Figure 1 : Pourcentage du type de transformation du riz selon la région du Bénin et le genre.

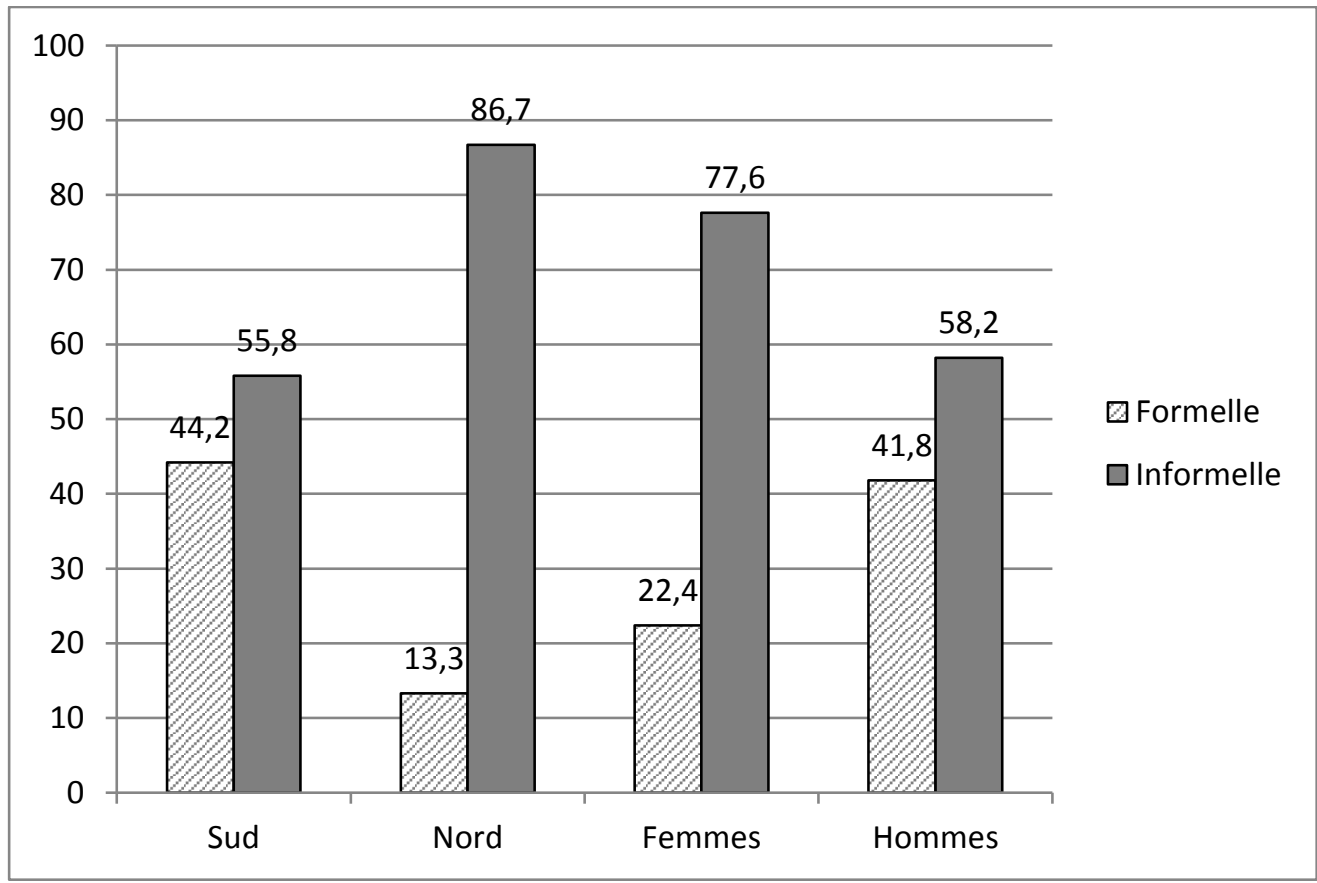

Figure 2 : Pourcentages des sources d'apprentissage sur la transformation du riz selon la région du Bénin et le genre. 
Tableau 3 : Pourcentages des transformateurs du riz paddy en riz blanc et étuvé selon leurs pratiques en 2010, 2012 et 2018 dans les villages contrôles, radio et FFV.

\begin{tabular}{llllll}
\hline $\begin{array}{l}\text { Type de } \\
\text { riz }\end{array}$ & $\begin{array}{l}\text { Approche } \\
\text { d'apprentissage }\end{array}$ & Année & $\begin{array}{l}\text { Pratiques non } \\
\text { recommandées }\end{array}$ & $\begin{array}{l}\text { Pratiques peu } \\
\text { recommandées }\end{array}$ & $\begin{array}{l}\text { Pratiques } \\
\text { recommandées }\end{array}$
\end{tabular}

\begin{tabular}{|c|c|c|c|c|c|}
\hline & & 2010 & $77,8^{\mathrm{a}}$ & $22,2^{\mathrm{a}}$ & $0,0^{\mathrm{a}}$ \\
\hline & Controle & 2012 & $30,6^{\mathrm{b} \alpha}$ & $55,6^{\text {b } \alpha}$ & $13,8^{\mathrm{b} \alpha}$ \\
\hline \multirow{11}{*}{$\begin{array}{l}\text { Riz } \\
\text { blanc }\end{array}$} & & 2018 & $28,3^{b^{8}}$ & $53,3^{\text {b } \alpha}$ & $18,4^{\text {ba }}$ \\
\hline & & 2010 & $86,4^{\mathrm{c}}$ & $13,6^{\mathrm{c}}$ & $0,0^{\mathrm{c}}$ \\
\hline & Radio & & & & \\
\hline & & 2012 & $31,9^{\mathrm{d} \beta}$ & $45,4^{\mathrm{d} \beta}$ & $22,7^{\mathrm{d} \beta}$ \\
\hline & & 2018 & $27,0^{\mathrm{d} \beta}$ & $47,6^{\mathrm{d} \beta}$ & $25,4^{\mathrm{d} \beta}$ \\
\hline & & 2010 & $69,6^{\mathrm{e}}$ & $28,6^{\mathrm{e}}$ & $1,8^{\mathrm{e}}$ \\
\hline & FFV & & & & \\
\hline & & 2012 & $1,8^{\mathrm{f}^{\mathrm{s}}}$ & $37,5^{f^{5}}$ & $60,7^{f^{8}}$ \\
\hline & & 2018 & $1,8^{\mathrm{f}^{\natural}}$ & $34,6^{\mathrm{f}^{5}}$ & $63,6^{\mathrm{f}^{8}}$ \\
\hline & & 2010 & $100,0^{\mathrm{a}}$ & $0,0^{\mathrm{a}}$ & $0,0^{\mathrm{a}}$ \\
\hline & & 2012 & $31.1^{\mathrm{b} \alpha}$ & $60,0^{\mathrm{b} \alpha}$ & $8,9^{\mathrm{ba}}$ \\
\hline \multirow{9}{*}{$\begin{array}{l}\text { Riz } \\
\text { étuvé }\end{array}$} & & 2018 & $34,1^{b \alpha}$ & $56,8^{\text {b } \alpha}$ & $9,1^{\mathrm{b} \alpha}$ \\
\hline & & 2010 & $100,0^{\mathrm{c}}$ & $0,0^{\mathrm{c}}$ & $0,0^{\mathrm{c}}$ \\
\hline & Radio & & & & \\
\hline & & 2012 & $0,0^{\mathrm{d} \beta}$ & $25,5^{\mathrm{d} \beta}$ & $74,5^{\mathrm{d} \beta}$ \\
\hline & & 2018 & $0,0^{\mathrm{d} \beta}$ & $24,0^{\mathrm{d} \beta}$ & $76,0^{\mathrm{d} \beta}$ \\
\hline & & 2010 & $92,3^{\mathrm{e}}$ & $7,7^{\mathrm{e}}$ & $0,0^{\mathrm{e}}$ \\
\hline & FFV & & & & \\
\hline & & 2012 & $0,0^{\mathrm{f} \beta}$ & $23,1^{\mathrm{f}^{6}}$ & $76,9^{\mathrm{f}^{5}}$ \\
\hline & & 2018 & $0,0^{\mathrm{fB}}$ & $19,6^{\mathrm{f}^{8}}$ & $80,4^{\mathrm{f}^{8}}$ \\
\hline
\end{tabular}

Note : Différentes lettres en exposants des valeurs des caractéristiques au niveau d'un même type de riz et d'une même approche d'apprentissage entre '2010 et 2012', '2010 et 2018' et '2012 et 2018' expriment une différence significative à 5\% avec le test de Wilcoxon.

Différents symboles en exposants des valeurs des caractéristiques au niveau d'une même colonne en 2012 et en 2018 expriment une différence significative à 5\% avec le test ANOVA à un facteur (LSD). 
Tableau 4 : Déterminants de l'amélioration des pratiques de transformation du paddy en riz blanc.

\begin{tabular}{|c|c|c|c|}
\hline Variables indépendantes & Coefficient & $\begin{array}{l}\text { Erreur } \\
\text { standard }\end{array}$ & Probabilité \\
\hline Apprentissage par la FFV & 0,366 & 0,081 & $0,000 * * *$ \\
\hline Apprentissage par la radio & 0,066 & 0,079 & 0,403 \\
\hline Ethnie & 0,116 & 0,094 & 0,218 \\
\hline Genre & $-0,132$ & 0,092 & 0,152 \\
\hline Age & $-0,002$ & 0,003 & 0,604 \\
\hline Situation matrimoniale & $-0,098$ & 0,130 & 0,449 \\
\hline Nombre de personnes dans le ménage & 0,002 & 0,009 & 0,779 \\
\hline Niveau d'instruction & $-0,022$ & 0,039 & 0,574 \\
\hline Religion & 0,039 & 0,032 & 0,228 \\
\hline Principale activité en 2010 & $-0,028$ & 0,082 & 0,728 \\
\hline Durée de l'activité au cours de l'année & $-0,122$ & 0,051 & $0,018 * *$ \\
\hline Nombre d'années d'expérience & 0,009 & 0,005 & 0,109 \\
\hline Type de transformation en 2010 & $-0,0254$ & 0,047 & 0,595 \\
\hline Niveau d'accessibilité au riz paddy & 0.021 & 0,068 & 0,753 \\
\hline Fréquence de rencontre avec les facilitateurs & 0,153 & 0,064 & $0,016^{* *}$ \\
\hline Pratiques de transformation en 2010 & 0,026 & 0,023 & 0,254 \\
\hline Participation à une formation en étuvage & $-0,065$ & 0,128 & 0,608 \\
\hline Possession d'un téléphone portable en 2010 & 0,098 & 0,090 & 0,279 \\
\hline Accès au crédit formel en 2010 & 0,136 & 0,071 & $0,056^{*}$ \\
\hline Accès au crédit informel en 2010 & 0,074 & 0,072 & 0,305 \\
\hline Accès au crédit informel & 0,187 & 0,098 & $0,057^{*}$ \\
\hline Constante & 1,323 & 0,302 & 0,000 \\
\hline \multicolumn{4}{|l|}{ log likelihood $=-406,380$} \\
\hline Nombre d'observations $=188$ & & & \\
\hline
\end{tabular}


Tableau 5 : Déterminants de l'amélioration des pratiques d'étuvage du riz.

\begin{tabular}{|c|c|c|c|}
\hline Variables indépendantes & Coefficient & $\begin{array}{l}\text { Erreur } \\
\text { standard }\end{array}$ & Probabilité \\
\hline Apprentissage par la FFV & 1,054 & 0,1167 & $0,000 * * *$ \\
\hline Apprentissage par la radio & 0,809 & 0,107 & $0,000 * * *$ \\
\hline Ethnie & $-0,212$ & 0,179 & 0,237 \\
\hline Genre & $-0,194$ & 0,499 & 0,697 \\
\hline Age & 0,001 & 0,004 & 0,926 \\
\hline Situation matrimoniale & 0,031 & 0,084 & 0,708 \\
\hline Nombre de personnes dans le ménage & 0,007 & 0,011 & 0,501 \\
\hline Niveau d'instruction & 0,007 & 0,063 & 0,908 \\
\hline Religion & 0,126 & 0,135 & 0,351 \\
\hline Principale activité en 2010 & 0,072 & 0,068 & 0,294 \\
\hline Durée de l'activité au cours de l'année & 0,120 & 0,114 & 0,292 \\
\hline Nombre d'années d'expérience & 0,003 & 0,004 & 0,445 \\
\hline Type de transformation en 2010 & 0,258 & 0,093 & $0,006 * * *$ \\
\hline Niveau d'accessibilité au riz paddy & 0,027 & 0,069 & 0,687 \\
\hline Fréquence de rencontre avec les facilitateurs & 0,048 & 0,086 & 0,580 \\
\hline Pratiques d'étuvage en 2010 & 0,029 & 0,046 & 0,518 \\
\hline Participation à une formation en étuvage & 0,030 & 0,142 & 0,830 \\
\hline Possession d'un téléphone portable en 2010 & $-0,333$ & 0,355 & 0,348 \\
\hline Accès au crédit formel en 2010 & $-0,014$ & 0,072 & 0,837 \\
\hline Accès au crédit informel en 2010 & 0,053 & 0,087 & 0,542 \\
\hline Accès au crédit informel & $-0,084$ & 0,133 & 0,525 \\
\hline Constante & 0,645 & 0,817 & 0,430 \\
\hline \multicolumn{4}{|l|}{$\log$ likelihood $=-271,56672$} \\
\hline Nombre d'observations $=125$ & & & \\
\hline
\end{tabular}


Tableau 6 : Caractéristiques physiques des échantillons de riz blanc et étuvé.

\begin{tabular}{|c|c|c|c|c|c|c|c|c|}
\hline $\begin{array}{l}\text { Type } \\
\text { de riz }\end{array}$ & $\begin{array}{l}\text { Approch } \\
\text { d'apprer }\end{array}$ & ssage & $\begin{array}{l}\text { \% } \\
\text { impuretés }\end{array}$ & $\begin{array}{l}\text { Taux } \\
\text { de } \\
\text { brisure }\end{array}$ & $\begin{array}{l}\text { Forme } \\
\text { (L/l) }\end{array}$ & $\begin{array}{l}\text { Dureté } \\
(\mathbf{K g})\end{array}$ & $\begin{array}{l}\text { Cour } \\
\text { blanc }\end{array}$ & Couleur \\
\hline \multirow{6}{*}{$\begin{array}{l}\text { Riz } \\
\text { blanc }\end{array}$} & Contrôle & 2010 & $2,24^{\mathrm{a}}$ & $0,29^{\mathrm{b}}$ & $3,21^{\mathrm{b}}$ & $5,79^{\mathrm{b}}$ & $1,46^{\mathrm{b}}$ & - \\
\hline & & 2012 & $1,59^{\mathrm{b} \alpha}$ & $0,56^{\mathrm{b} \alpha}$ & $3,15^{\mathrm{b} \alpha}$ & $8,27^{\mathrm{a} \alpha}$ & $0,96^{\mathrm{b} \alpha}$ & $67,39^{\alpha}$ \\
\hline & Radio & 2010 & $2,15^{\mathrm{c}}$ & $0,32^{\mathrm{d}}$ & $3,01^{\mathrm{d}}$ & $6,07^{\mathrm{d}}$ & $0,79^{\mathrm{d}}$ & - \\
\hline & & 2012 & $1,27^{\mathrm{d} \alpha}$ & $0,52^{\mathrm{d} \alpha}$ & $3,11^{\mathrm{d} \alpha}$ & $8,53^{\mathrm{c} \alpha}$ & $0,89^{\mathrm{d} \alpha}$ & $68,06^{\alpha}$ \\
\hline & FFV & 2010 & $1,55^{\mathrm{e}}$ & $0,28^{\mathrm{f}}$ & $3,57^{\mathrm{f}}$ & $5,58^{\mathrm{f}}$ & $1,44^{\mathrm{f}}$ & - \\
\hline & & 2012 & $0,68^{\mathrm{f} \alpha}$ & $0,49^{\mathrm{f} \alpha}$ & $3,28^{\mathrm{f} \alpha}$ & $8,79^{\mathrm{e} \alpha}$ & $1,48^{\mathrm{f} \alpha}$ & $68,33^{\alpha}$ \\
\hline \multirow{6}{*}{$\begin{array}{l}\text { Riz } \\
\text { étuvé }\end{array}$} & Contrôle & 2010 & $2,33^{b}$ & $0,18^{\mathrm{b}}$ & $3,26^{\mathrm{b}}$ & $11,42^{\mathrm{b}}$ & $0,04^{\mathrm{b}}$ & \\
\hline & & 2012 & $4,79^{\mathrm{a} \alpha}$ & $0,29^{\mathrm{a} \alpha}$ & $3,36^{\mathrm{b} \alpha}$ & $14,24^{\mathrm{a} \alpha}$ & $0,69^{\mathrm{b} \alpha}$ & $56,23^{\alpha}$ \\
\hline & Radio & 2010 & $2,01^{\mathrm{d}}$ & $0,23^{\mathrm{d}}$ & $3,07^{\mathrm{d}}$ & $12,38^{\mathrm{d}}$ & $0,87^{\mathrm{c}}$ & \\
\hline & & 2012 & $1,59^{\mathrm{d} \beta}$ & $0,15^{\mathrm{d} \beta}$ & $3,22^{\mathrm{d} \alpha}$ & $14,76^{\mathrm{c} \alpha}$ & $0,13^{\mathrm{d} \alpha}$ & $56,58^{\alpha}$ \\
\hline & FFV & 2010 & $1,12^{\mathrm{f}}$ & $0,20^{\mathrm{e}}$ & $3,20^{\mathrm{f}}$ & $12,46^{\mathrm{f}}$ & $0,10^{\mathrm{f}}$ & \\
\hline & & 2012 & $1,09^{\mathrm{f}^{\natural}}$ & $0,14^{\mathrm{f}^{\natural}}$ & $3,20^{\mathrm{f} \alpha}$ & $14,97^{\mathrm{e} a}$ & $0,09^{\mathrm{f} \alpha}$ & $57,19^{\alpha}$ \\
\hline
\end{tabular}

Note : Différentes lettres en exposants des valeurs des caractéristiques au niveau d'une même approche d'apprentissage entre 2010 et 2012 expriment une différence significative à 5\% avec le test "Student paired t-test"

Différents symboles en exposants des valeurs des caractéristiques au niveau d'une même colonne en 2012 expriment une différence significative à 5\% avec le test ANOVA à un facteur (LSD) pour la comparaison des caractéristiques en 2012.

Tableau 7 : Caractéristiques de cuisson des échantillons de riz blanc et étuvé.

\begin{tabular}{lllllll}
\hline $\begin{array}{l}\text { Type de } \\
\text { riz }\end{array}$ & $\begin{array}{l}\text { Approche } \\
\text { d'apprentissage }\end{array}$ & Elongation & $\begin{array}{l}\text { Absorption } \\
\text { d'eau }\end{array}$ & $\begin{array}{l}\text { Augmentation } \\
\text { de volume }\end{array}$ & $\begin{array}{l}\text { Temps de } \\
\text { cuisson }\end{array}$ \\
\hline \multirow{4}{*}{$\begin{array}{l}\text { Riz } \\
\text { blanc }\end{array}$} & Contrôle & 2010 & $1,38^{\mathrm{b}}$ & $1,75^{\mathrm{b}}$ & $2,76^{\mathrm{b}}$ & $16,67^{\mathrm{b}}$ \\
& & 2012 & $1,36^{\mathrm{b} \alpha}$ & $1,71^{\mathrm{b} \alpha}$ & $3,28^{\mathrm{a} \alpha}$ & $18,44^{\mathrm{av}}$ \\
\cline { 2 - 7 } & Radio & 2010 & $1,39^{\mathrm{d}}$ & $1,64^{\mathrm{d}}$ & $2,56^{\mathrm{d}}$ & $16,5^{\mathrm{d}}$ \\
& & 2012 & $1,38^{\mathrm{d} \alpha}$ & $1,71^{\mathrm{d} \alpha}$ & $2,99^{\mathrm{d} \beta}$ & $18,83^{\mathrm{c} \alpha}$ \\
\hline & FFV & 2010 & $1,41^{\mathrm{f}}$ & $1,92^{\mathrm{f}}$ & $1,91^{\mathrm{f}}$ & $17,28^{\mathrm{f}}$ \\
& & 2012 & $1,41^{\mathrm{f} \alpha}$ & $2,20^{\mathrm{f} \alpha}$ & $2,85^{\mathrm{e}^{\mathrm{b}}}$ & $19,33^{\mathrm{e} \alpha}$ \\
\hline \multirow{4}{*}{$\begin{array}{l}\text { Riz } \\
\text { étuvé }\end{array}$} & Contrôle & 2010 & $1,34^{\mathrm{b}}$ & $1,64^{\mathrm{b}}$ & $3,23^{\mathrm{b}}$ & $19,94^{\mathrm{b}}$ \\
& & 2012 & $1,29^{\mathrm{b} \alpha}$ & $1,54^{\mathrm{b} \alpha}$ & $3,39^{\mathrm{b} \alpha}$ & $20,00^{\mathrm{b} \alpha}$ \\
\cline { 2 - 7 } & Radio & 2010 & $1,38^{\mathrm{d}}$ & $1,67^{\mathrm{d}}$ & $3,03^{\mathrm{d}}$ & $20,44^{\mathrm{d}}$ \\
& & 2012 & $1,38^{\mathrm{d} \beta}$ & $1,70^{\mathrm{d} \alpha}$ & $3,12^{\mathrm{d} \alpha}$ & $21,39^{\mathrm{d} \alpha}$ \\
\hline & FFV & 2010 & $1,30^{\mathrm{f}}$ & $1,64^{\mathrm{f}}$ & $3,13^{\mathrm{f}}$ & $19,22^{\mathrm{f}}$ \\
& & 2012 & $1,36^{\mathrm{e}}$ & $1,80^{\mathrm{f} \alpha}$ & $3,10^{\mathrm{f} \alpha}$ & $20,00^{\mathrm{f} \alpha}$ \\
\hline
\end{tabular}

Note : Différentes lettres en exposants des valeurs des caractéristiques au niveau d'une même approche d'apprentissage entre 2010 et 2012 expriment une différence significative à $5 \%$ avec le test "Student paired t-test",

Différents symboles en exposants des valeurs des caractéristiques au niveau d'une même colonne en 2012 expriment une différence significative à 5\% avec le test ANOVA à un facteur (LSD) pour la comparaison des caractéristiques en 2012. 


\section{DISCUSSION}

Les résultats de l'étude ont montré que les femmes sont majoritaires (67\%) dans la transformation du riz comparativement aux hommes. Ce constat a été mis en évidence par Norman et Kebe (2006) qui ont montré que les femmes étaient plus responsables des travaux pénibles liés à la post-récolte du riz en Afrique. L'activité principale des femmes est le petit commerce ; ce qui corrobore les études de Fafchamps et al. (2005) qui ont montré l'importance du petit commerce pour les femmes rurales en Afrique subsaharienne.

La forte présence des femmes dans les sources informelles d'apprentissage agricole confirme les résultats de World Bank (2008) et Zossou et al. (2017) qui ont fait ressortir les contraintes socio-culturelles auxquelles sont confrontées les femmes rurales en Afrique Sub-saharienne dans la communication avec les hommes hors de leurs familles ; car la majorité des agents de vulgarisation sont de sexe masculin (World Bank, 2008 ; Katungi et al., 2008).

La prédominance des pratiques non recommandées au niveau des transformateurs en 2010 avant l'apprentissage montre que les groupes ciblés pour l'apprentissage social sont réellement dans le besoin de connaissances. $\mathrm{Ce}$ besoin manifeste des potentiels apprenants est un facteur important pouvant favoriser l'apprentissage collectif selon Leeuwis et van den Ban (2004). Les innovations locales faites par les transformateurs de riz suite à l'apprentissage par la vidéo et la radio confirment les résultats de Zossou et al. (2009a, 2009b) au centre du Bénin qui ont montré que l'apprentissage de l'étuvage du riz par la FFV a déclenché des innovations locales. Ces résultats montrent que les communautés agricoles disposent d'énormes connaissances endogènes adaptées à leurs réalités socioéconomiques et culturelles qui doivent être valorisées pour une durabilité de l'agriculture en Afrique Subsaharienne (Leeuwis et van den Ban, 2004 ; Zossou et al., 2017 ; Hinnou et al., 2021).

Le modèle de régression logistique de Poisson a fait ressortir quatre facteurs qui ont eu un effet positif significatif sur l'amélioration des pratiques de transformation du riz paddy en riz blanc; et trois facteurs qui ont eu un effet positif significatif sur l'étuvage du riz. L'apprentissage collectif par la vidéo FFV améliore les pratiques des transformateurs du riz. Ce résultat est conforme à d'autres études antérieures sur l'impact de l'apprentissage par la vidéo en milieu rural en Inde (Vidya et al., 2010) ; au Bénin (Zossou et al., 2009b ; Zoundji et al., 2018a) ; au Bangladesh (Van Mele et al., 2005 ; Chowdhury et al., 2011), au Ghana (David et Asamoah, 2011) ; et au Mali (Zoundji et al., 2018b). Dans un contexte d'insuffisance d'infrastructures technologiques et de faible niveau d'alphabétisation, l'utilisation de la vidéo dans la vulgarisation agricole est une opportunité (David et Asamoah, 2011). L'apprentissage collectif par la FFV réduit le problème de biais lié au genre car la chance est donnée aussi bien aux hommes et aux femmes de recevoir l'information au même moment.

L'apprentissage par la radio rurale est également un facteur déterminant dans l'amélioration des pratiques d'étuvage ; mais avec un effet moins important que celui observé dans le cas de la FFV. L'impact positif de la radio sur les pratiques post-récoltes du riz est conforme aux études de Hambly et Atibila (2003) au Ghana qui ont montré l'influence positive de la radio sur la productivité agricole dans les zones rurales pauvres. L'utilisation de ce canal de communication pour la vulgarisation agricole permet en effet de combler le fossé entre paysans, vulgarisateurs et chercheurs dans un contexte de forte ruralité et de fort taux d'illettrés (Chapman et al., 2003). 
La durée de l'activité au cours de l'année a un effet négatif significatif sur l'amélioration des pratiques des transformateurs. Ceci peut s'expliquer par la masse de travail qui est plus élevée quand les activités post-récoltes s'étalent sur toute l'année. Ce qui rend le transformateur du riz moins performant sur certains points essentiels tels que le triage du paddy, le battage à un endroit propre et le séchage au soleil puis à l'ombre.

La fréquence de rencontre avec les facilitateurs du développement rural a un effet positif sur l'amélioration des pratiques. Ces rencontres constituent en effet des opportunités d'échanges et d'apprentissage. Mais malheureusement l'effectif de ces facilitateurs est limité et les contraintes financières font qu'ils ne peuvent pas être en contact régulier avec tous ceux qui l'auraient souhaité dans le monde rural (Zossou et al., 2017).

L'accès au crédit a un effet positif significatif sur l'amélioration des pratiques. Ceci peut se justifier par le fait que l'accès au crédit améliore le capital financier (Zossou et al., 2020 ; Adekambi et al., 2021) qui par conséquent a un effet positif sur l'investissement en matériel et en maind'œuvre pour l'amélioration de la qualité du riz transformé.

Les transformateurs de riz qui font à la fois le riz étuvé et le riz blanc ont tendance à avoir de bonnes pratiques pour l'étuvage du riz en comparaison avec ceux qui font uniquement le riz étuvé. Ceci peut s'expliquer par le fait que la plupart de ceux qui font à la fois le riz étuvé et le riz blanc sont des producteurs de riz qui utilisent leur propre production de paddy comme matière première ; ce qui évite les inconvénients liés au mélange variétal et améliore donc les pratiques des transformateurs.

La qualité physique du riz étuvé (en termes de baisse d'impuretés) s'est considérablement améliorée dans les villages FFV et radio. Selon Fofana et al. (2011), la qualité physique des grains de riz tend à s'améliorer avec la diminution du pourcentage d'impuretés. Cette amélioration est probablement due à l'adoption des bonnes pratiques de battage, de triage, de vannage et de lavage suite à l'apprentissage collectif par la FFV et la radio.

L'amélioration de la qualité du riz étuvé due à la diminution du taux de brisure au décorticage est probablement due à l'adoption de bonnes pratiques sur la pré-cuisson du paddy à la vapeur et le séchage au soleil puis à l'ombre suite à l'apprentissage par la FFV. Ces bonnes pratiques sont des facteurs importants qui selon Houssou et al. (2004) réduisent significativement le taux de brisure au décorticage. L'amélioration de la dureté des grains de riz suite à l'apprentissage par la vidéo et la radio est un élément très important, surtout pour le stockage. Plus la dureté est élevée, plus les grains sont résistants aux attaques des insectes et moins sensibles au développement de moisissures (Fofana et al., 2011).

L'apparence des grains de riz est en grande partie déterminée par la translucidité de l'endoderme qui est inversement liée à la présence de cœurs blancs (Khush et al., 1979 ; Fofana et al., 2011). Le faible score observé après l'apprentissage par la vidéo et la radio en cœurs blancs prouve que la qualité du riz s'est améliorée car une forte proportion de cœurs blancs dégrade la qualité physique du riz et affecte négativement sa valeur marchande (Adu-Kwarten et al., 2003 ; Gayin et al., 2009). La couleur des grains de riz est meilleure dans les villages vidéo et radio comparativement à ce qui est observé dans les villages contrôles. La couleur du riz est déterminante dans le choix des consommateurs (Zossou et al., 2009b ; Demont et al., 2012). Plus le score de la couleur est élevé, plus la blancheur est nette 
(Hunterlab, 2008), et plus le consommateur est attiré par le produit final.

L'amélioration des pratiques d'étuvage du riz suite à l'apprentissage par la vidéo et la radio a eu un impact positif sur la qualité du riz en termes de l'élongation des grains et de l'augmentation de volume. L'élongation du riz est un facteur important dans le choix des consommateurs de riz au Bénin (Zossou et al., 2009b ; Demont et al., 2012). Plus le grain de riz est long, plus il est apprécié positivement par les consommateurs. A part les pratiques d'étuvage, d'autres facteurs liés à la variété du riz, aux conditions de culture et à l'écologie peuvent également influencer l'élongation des grains de riz (Fofana et al., 2011). Ces paramètres n'ont pas été pris en compte au cours de cette étude et limitent donc les conclusions tirées. L'augmentation de volume constitue également un facteur important dans le choix des consommateurs de riz au Bénin (Zossou et al., 2009b ; Demont et al., 2012).

\section{Conclusion}

L'apprentissage collectif par la vidéo 'Farmer to Farmer Vidéo' (FFV) et la radio rurale a favorisé l'amélioration des pratiques des petits transformateurs du riz local au Bénin. L'apprentissage des petits exploitants agricoles dans une approche d'école inclusive a également permis de développer leur esprit créatif pour le développement et l'utilisation d'innovations locales qui traduisent mieux leurs réalités. L'apprentissage par la FFV paraît plus efficace car il est à la fois audio et visuel et donc plus illustratif. De plus, les acteurs de la vidéo sont les pairs des petits exploitants agricoles; ce qui renforce le climat de confiance et stimule des échanges interactifs pour un apprentissage collectif et inclusif. L'apprentissage par la radio doit par contre être accompagné d'un renforcement de capacités des animateurs afin que l'information délivrée soit pertinente et de bonne qualité. Les résultats de cette étude montrent que certaines caractéristiques physiques qui constituent des éléments de choix pour les consommateurs de riz au Bénin se sont améliorées suite principalement à l'apprentissage par la vidéo. Les améliorations observées au niveau de la qualité du riz confirment les bonnes pratiques adoptées par les transformateurs de riz suite principalement à l'apprentissage collectif par la vidéo. Cependant, plus d'investigations sont nécessaires pour une maîtrise des paramètres comme la variété, l'écologie et les méthodes de culture afin de mieux accompagner les acteurs de la transformation du riz local. Cette recherche confirme le rôle important que joue l'apprentissage collectif par la vidéo et la radio dans le renforcement des systèmes d'innovation agricole en Afrique subsaharienne caractérisée par la prédominance de petits producteurs orientés plus vers une agriculture familiale de subsistance.

\section{CONFLITS D'INTÉRÊTS}

Les auteurs déclarent qu'il n'y a aucun conflit d'intérêts

\section{CONTRIBUTIONS DES AUTEURS}

Tous les auteurs ont participé à l'élaboration du protocole, l'analyse des données, la rédaction du projet de manuscrit et la correction $\mathrm{du}$ manuscrit. L'ordre d'apparition des auteurs est bien conforme aux diverses contributions faites.

\section{REMERCIEMENTS}

Les auteurs adressent leur sincères remerciements au Centre du Riz pour l'Afrique qui a contribué au financement de la recherche. Ils remercient également tous les acteurs de la transformation du riz local au Bénin pour leur disponibilité et les informations fournies. Les auteurs tiennent également à remercier tous les 
lecteurs externes qui ont contribué à l'amélioration de la qualité du manuscrit.

\section{RÉFÉRENCES}

Adu-Kwarten E, Ellis WO, Oduro I, Manful JT. 2003. Rice grain quality: a comparison of local varieties with new varieties under study in Ghana. Food Control, $14 \quad$ (7): 507-514. https://doi.org/10.1016/S0956-

7135(03)00063-X

Adekambi S, Codjovi JEA, Yabi JA. 2021. Facteurs déterminants l'adoption des mesures de gestion intégrée de la fertilité des sols (GIFS) au nord du Bénin : une application du modèle probit multivarié au cas de producteurs de maïs. Int. J. Biol. Chem. Sci., 15 (2): 664-678. DOI : https://dx.doi.org/10.4314/ijbcs.v15i2.22

Axinn HG. 1997. Challenges to agricultural extension in the twenty first century. In Farmer-led Extension: Concepts andPpractices, Scarborough V, Killough S, Johnson DA, Farrington J (eds). Londres, UK: Intermediate Technology Publications Ltd; 13-21.

Chapman R, Blench R, Kranjac-Berisavljevic G, Zakariah ABT. 2003. Rural Radio in Agricultural Extension: the example of vernacular radio programmes on soil and water conservation in northern Ghana. Overseas Development Institute: Londres, UK.

Chowdhury AH, Van Mele P, Hauser M. 2011. Contribution of farmer-to-farmer video to capital assets building: evidence from Bangladesh. Journal of Sustainable Agriculture, 35 (4): 408-435. DOI: 10.1080/10440046.2011.562059

David S, Asamoah A. 2011. Video as a tool for agricultural extension in Africa: a case study from Ghana. International Journal of Education and Development using
Information and Communication Technology, 7 (1): 26-41.

Delarue J, Cochet H. 2011. Proposition méthodologique pour l'évaluation des projets de développement : L'évaluation systémique d'impact. Economie Rurale, 3 (323):

36-54.DOI :

https://doi.org/10.4000/economierurale.3 034

Demont M, Zossou E, Rutsaert P, Ndour M, Van Mele P, Verbeke W. 2012. Consumer valuation of improved rice parboiling technologies in Benin. Food Quality and Preference, 23 (1): 63-70. DOI: 10.1016/j.foodqual.2011.07.005

Fafchamps M, Gabre-Madhin E, Minten B. 2005. Increasing returns and market efficiency in agricultural trade. Journal of Development Economics, 78 (2) : 406442.

DOI:

10.1016/j.jdeveco.2004.10.001

FAO. 1997. Elaboration d'un plan national de relance de la filière riz. Rapport définitif, volumes 1 et 2 , FAO/Projet TCP/BEN/5613 (A). Cotonou, Bénin.

Fofana M, Wanvoeke J, Manful J, Futakuchi K, Van Mele P, Zossou E, Bléoussi TMR. 2011. Effect of improved parboiling methods on the physical and cooked grain characteristics of rice varieties in Benin. International Food Research Journal, 18 (2): 697-703.

Gayin J, Manful JT, Johnson PNT. 2009. Rheological and sensory properties of rice varieties from improvement programme in Ghana. International Food Research Journal, (16): 167-174.

Hakiza JJ, Odogola W, Mugisha J, Semana AR, Nalukwago I, Okoth J, Ekwamu A. 2004. Challenges and Prospects of Disseminating Technologies through Farmer Field Schools: Lessons Learnt Based on Experience from Uganda. Uganda Journal of Agricultural Sciences, 
9:

163-175.

DOI:

http://dx.doi.org/10.4314/jae.v15i2.8

Hambly OH, Atibila J. 2003. Linking agricultural research and rural radio in Africa: new opportunities for communicating innovation and experiences from Northern Ghana. A case study for the CTA ICT Observatory 2003: ICTs - Transforming Agricultural Extension? International Service for National Agricultural Research: The Hague, The Netherlands.

Hinnou CL, Agbotridja VD, Ahoyo Adjovi RN. 2021. Analyse des besoins en mécanisation agricole basée sur les logiques paysannes dans les pôles de développement agricole du Bénin. Int. $J$. Biol. Chem. Sci., 15 (2): 536-549. DOI : https://dx.doi.org/10.4314/ijbcs.v15i2.13

Houssou P, Singbo A, Amonson E. 2004. Influence des modes de séchage du paddy sur la qualité du riz au sud du Bénin. Rapport d'activité INRAB: Cotonou, Bénin.

Houssou P. 2005. Development and test of improved parboiling equipment for paddy rice in Benin. Rapport PTAA, INRAB: Porto-Novo, Bénin.

Hunterlab. 2008. Hunter L,a,b Color Scale. http://www.hunterlab.com/appnotes/an0 8_96a.pdf.

Juliano BO. 1994. Le riz dans la Nutrition Humaine. FAO: Rome, Italie.

Katungi E, Edmeades S, Smale M. 2008. Gender, social capital and information exchange in rural Uganda. Journal of International Development, 20 (1): 3552. DOI: https://doi.org/10.1002/jid.1426

Khush GS, Paule CM, De la Cruz NM. 1979. Rice grain quality evaluation and improvement. In: Proceedings of workshop on chemical aspects of rice grain quality, International Rice Research
Institute (ed). IRRI: Manila, Philippines; 21-31.

Leeuwis C, van den Ban A. 2004. Communication for Rural Innovation: Rethinking Agriculture Extension (3rd edn). Blackwell Publishing: Oxford, UK.

Mancini F, Van Bruggen, AHC, Jiggins JLS. 2007. Evaluating cotton integrated pest management (IPM) farmer field school outcomes using the sustainable livelihoods approach in India. Experimental Agriculture, 43 (1): 97112.

DOI: https://doi.org/10.1017/S0014479706004 $25 \mathrm{X}$

Nederlof ES, Odonkor EN. 2006. Lessons from an Experiential Learning Process: The Case of Cowpea Farmer Field Schools in Ghana. Journal of Agricultural Education and Extension, 12 (4): 249-271. https://doi.org/10.1080/13892240601062 447

Norman JC, Kebe B. 2006. African smallholder farmers: rice production and sustainable livelihoods. International Rice Commission Newsletter, 55: 33-42. DOI: $\quad$ http://www.fao.org/3/aa0869t/a0869t02.pdf

Pretty JN, Vodouhe DS. 1997. Using rapid or participatory rural appraisal. In Improving Agricultural Extension: a Reference Manual, Swanson BE, Bentz RP, Sofranko AJ (eds). FAO: Rome, Italie; 47-55.

Ramírez R, Quarry W. 2004. Communication strategies in the age of decentralisation and privatization of rural services: lessons from two African experiences. Agricultural Research and Extension Network Paper (136): 1-20.

Roy P, Orikasa T, Okadome H, Nakamura N, Shiina T. 2011. Processing Conditions, Rice Properties, Health and Environment. International Journal of Environmental 
Research and Public Health, 8 (6): $1957-$ 1976. DOI: 10.3390/ijerph8061957

Struif-Bontkes TE, Wopereis MCS, Ankou K, Lamboni D. 2003. The use of QUEFTS in search of balanced fertilizer recommendations for maize in Togo. In Decision Support Tools for Smallholder Agriculture in sub-Saharian Africa: a Practical Guide, Wopereis MCS, StruifBontkes TE (eds). IFDC, CTA: Wageningen, The Netherlands; 68-84.

Van Mele P, Salahuddin A, Magor NP. 2005. Innovations in Rural Extension: Case Studies from Bangladesh. CABI Publishing: Wallingford, UK.

Van Mele P. 2006. Zooming-in, zooming-out: A novel method to scale up local innovations and sustainable technologies. International Journal of Agricultural Sustainability, 4 (2): 131-142. DOI: 10.1080/14735903.2006.9684796

Vidya P, Manivannan C, Sudeep Kumar NK. 2010. Effectiveness of an educational interactive video-dvd on dairy health management practices in terms of knowledge gain among dairy farmers. The Online Journal of Rural Research and Policy, 5 (7): 1-17. DOI: 10.4148/ojrrp.v5i7.267

World Bank. 2008. World development Report 2008. The World Bank: Washington, USA.

Zossou E, Van Mele P, Vodouhe SD, Wanvoeke J. 2009a. Comparing Farmer to Farmer video with workshops to train rural women in improved rice parboiling in Central Benin. Journal of Agricultural Education and Extension, 15 (4): 329 339. DOI: $10.1080 / 13892240903309561$ Zossou E, Van Mele P, Vodouhe SD, Wanvoeke J. 2009b. The power of video to trigger innovation : rice processing in Central Benin. International Journal of Agricultural Sustainability, 7 (2): 119129. DOI: $10.3763 /$ ijas.2009.0438

Zossou E, Vodouhe DS, Van Mele P, AgbohNoameshie AR, Lebailly Ph. 2015. Linking local rice processors' access to rural radio, gender, and livelihoods in Benin. Development in Practice, 25 (7): 1057-1066.

DOI:

10.1080/09614524.2015.1069261

Zossou E, Arouna A, Aliou A, AgbohNoameshie AR. 2017. Gender gap in acquisition and practice of agricultural knowledge: Case study of rice farming in West Africa. Experimental Agriculture, 53 (4): 566-577. DOI: 10.1017/S0014479716000582

Zoundji CG, Okry F, Vodouhê DS, Bentley JW. 2018a. Towards sustainable vegetable growing with farmer learning videos in Benin. International Journal of Agricultural Sustainability, 16 (1): 54-63. DOI: 10.1080/14735903.2018.1428393

Zoundji CG, Vodouhê DS, Okry F, Bentley JW, Tossou RC. 2018b. Beyond Striga Management: Learning Videos Enhanced Farmers' Knowledge on Climate-Smart Agriculture in Mali. Sustainable Agriculture Research, 7 (1): 80-91. DOI: 10.5539/sar.v7n1p80. 